\title{
Apolipoprotein E-containing high-density lipoprotein (HDL) cholesterol is a better indicator for anti-atherosclerotic function of HDL than total HDL cholesterol: a community-based cohort study
}

\author{
Yue Qi, M.D., Ph.D., Jing Liu, M.D., Ph.D., Miao Wang, M.D., Jiayi Sun, M.D., Jun Liu, M.D., Dong Zhao, M.D., Ph.D. \\ Department of Epidemiology, Beijing An Zhen Hospital, Capital Medical University, \\ Beijing Institute of Heart, Lung and Blood Vessel Diseases, \\ The Key Laboratory of Remodeling-related Cardiovascular Diseases, Ministry of Education, Beijing, China.
}

\begin{abstract}
Aim: APOE-containing HDL (APOE-HDL) particle may be necessary to stimulate the reverse cholesterol transport process, and was associated with CHD reported by either underpowered or hospitalbased studies. We assessed that whether APOE-HDL cholesterol is causally associated with atherosclerotic CVD risk and this association is independent of total HDL cholesterol.
\end{abstract}

Methods: APOE-HDL cholesterol and HDL cholesterol were measured in 5417 cardiovascular disease-free participants (women, 51.8\%) aged 45-79 years at baseline in the Chinese Multiprovincial Cohort Study. APOE-HDLC/HDLC ratio was calculated to assess the relative proportion of APOE-HDL cholesterol in total HDL cholesterol. Multivariate Cox regression was employed to assess the association between HDL related biomarkers and 10-year CHD incident risk.

Results: Mean levels of baseline APOE-HDL cholesterol and APOEHDLC/HDLC ratio were $5.15 \pm 1.60 \mathrm{mg} / \mathrm{dL}$ and $9.05 \pm 0.94$ $\%$, respectively. HDL cholesterol was strongly correlated with APOEHDL cholesterol (partial r: 0.978), and moderately with APOEHDLC/HDLC ratio (partial r: 0.604). Total of 100 incident CHD events occurred during 10-year follow-up. High levels of baseline HDL related biomarkers were significantly negatively associated with incident CHD risk. However, there were no overlaps in $95 \%$ confidence interval of absolute risk for the comparison of APOEHDLC/HDLC ratio $(0.48,95 \% \mathrm{CI}=0.44-0.52)$ with HDL cholesterol $(0.83,95 \% \mathrm{CI}=0.78-0.88)$ among participants with the highest level of these parameters. Furthermore, among participants with different levels of HDL cholesterol, baseline APOE-HDLC/HDLC ratio was negatively associated with incident CHD risk, but participants in the highest category of HDL cholesterol had the lowest HR associated with APOE-HDLC/HDLC ratio.

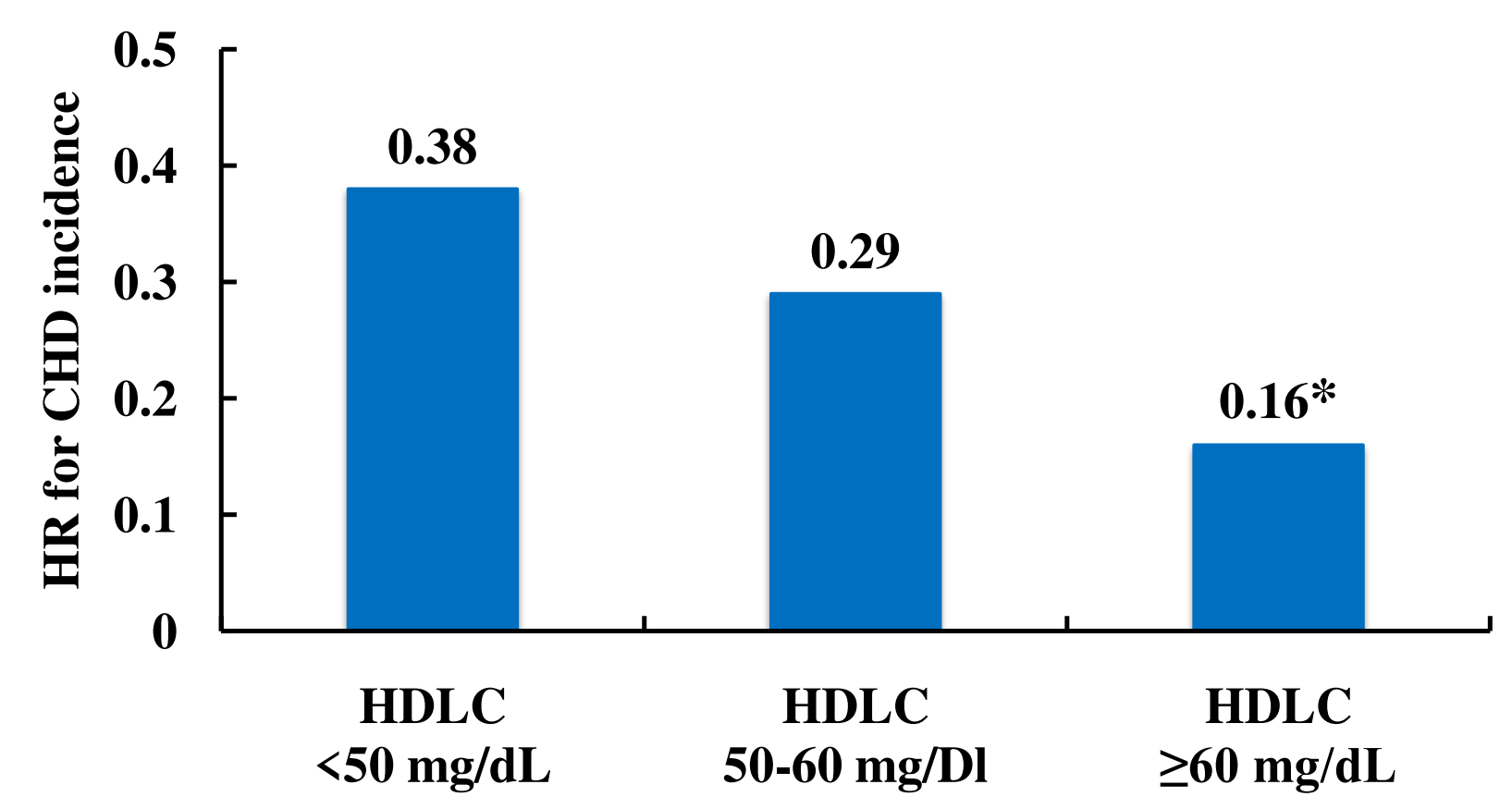

Figure. Cox regression analysis of APOE-HDLC/HDLC ratio associated with the risk of CHD incidence among HDLC subgroup
Conclusions: In conclusion, our findings revealed that APOEHDLC/HDLC ratio was significantly associated with 10-year increased risk of incident CHD, even beyond HDL cholesterol, suggesting that APOE containing HDL can serve as a better indicator for the antiatherosclerotic function of HDL than total HDL cholesterol.

Table. Cox regression analysis of HDL related biomarkers on the long-term risk of CHD incidence

\begin{tabular}{|c|c|c|c|c|}
\hline \multirow{2}{*}{ Clinical Features } & \multicolumn{4}{|c|}{ Subgroup of HDL related biomarkers } \\
\hline & $\begin{array}{c}\text { Group } \\
1\end{array}$ & Group 2 & Group 3 & Group 4 \\
\hline $\begin{array}{l}\text { APOE-HDLC/HDLC } \\
\text { ratio }(\%)\end{array}$ & $<8.6$ & $8.6-9.2$ & 9.3-9.9 & $\geq 10.0$ \\
\hline No. of participants & 1367 & 1628 & 1643 & 779 \\
\hline No. of CHD events & 29 & 33 & 30 & 8 \\
\hline $\begin{array}{l}\text { Incidence rate (per } \\
100,000 \text { person-years) }\end{array}$ & 342 & 290 & 273 & 129 \\
\hline $\begin{array}{l}\text { Multivariate adjusted } \\
\text { HR( } 95 \% \text { CI })\end{array}$ & Reference & $\begin{array}{c}0.98 \\
(0.55-1.75)\end{array}$ & $\begin{array}{c}0.84 \\
(0.48-1.46)\end{array}$ & $\begin{array}{c}0.26 \\
(0.10-0.71)\end{array}$ \\
\hline Absolute risk (\%) & $\begin{array}{c}4.18 \\
(3.96-4.36) \\
\end{array}$ & $\begin{array}{c}2.69 \\
(2.58-2.82) \\
\end{array}$ & $\begin{array}{c}2.45 \\
(2.35-2.59) \\
\end{array}$ & $\begin{array}{c}0.48 \\
(0.44-0.52) \\
\end{array}$ \\
\hline HDLC (mg/dl) & $\begin{array}{l}<40(\mathrm{M}) \\
<50(\mathrm{~F})\end{array}$ & $\begin{array}{l}40-49(\mathrm{M}) \\
50-59(\mathrm{~F})\end{array}$ & $\begin{array}{l}50-59(\mathrm{M}) \\
60-69(\mathrm{~F})\end{array}$ & $\begin{array}{l}\geq 60(\mathrm{M}) \\
\geq 70(\mathrm{~F})\end{array}$ \\
\hline No. of participants & 1056 & 1820 & 1370 & 1171 \\
\hline No. of CHD events & 31 & 34 & 23 & 12 \\
\hline $\begin{array}{l}\text { Incidence rate (per } \\
100,000 \text { person-years) }\end{array}$ & 415 & 273 & 244 & 156 \\
\hline $\begin{array}{l}\text { Multivariate adjusted } \\
\text { HR }(95 \% \text { CI })\end{array}$ & Reference & $\begin{array}{c}0.65 \\
(0.39-1.11)\end{array}$ & $\begin{array}{c}0.54 \\
(0.29-1.02)\end{array}$ & $\begin{array}{c}0.39 \\
(0.18-0.88)\end{array}$ \\
\hline Absolute risk (\%) & $\begin{array}{c}4.08 \\
(3.87-4.38) \\
\end{array}$ & $\begin{array}{c}2.36 \\
(2.20-2.46) \\
\end{array}$ & $\begin{array}{c}1.69 \\
(1.62-1.78) \\
\end{array}$ & $\begin{array}{c}0.83 \\
(0.78-0.88) \\
\end{array}$ \\
\hline APOE-HDLC (mg/dl) & $\begin{array}{l}<4.0(\mathrm{M}) \\
<5.0(\mathrm{~F})\end{array}$ & $\begin{array}{c}4.0-4.9(\mathrm{M}) \\
5.0-5.9(\mathrm{~F})\end{array}$ & $\begin{array}{l}5.0-5.9(\mathrm{M}) \\
6.0-6.9(\mathrm{~F})\end{array}$ & $\begin{array}{l}\geq 6.0(\mathrm{M}) \\
\geq 7.0(\mathrm{~F})\end{array}$ \\
\hline No. of participants & 1925 & 1483 & 990 & 1019 \\
\hline No. of CHD events & 46 & 31 & 13 & 10 \\
\hline $\begin{array}{l}\text { Incidence rate (per } \\
100,000 \text { person-years) }\end{array}$ & 355 & 302 & 193 & 142 \\
\hline $\begin{array}{l}\text { Multivariate adjusted } \\
\text { HR }(95 \% \text { CI })\end{array}$ & Reference & $\begin{array}{c}0.81 \\
(0.49-1.33)\end{array}$ & $\begin{array}{c}0.58 \\
(0.28-1.21)\end{array}$ & $\begin{array}{c}0.42 \\
(0.20-0.86)\end{array}$ \\
\hline Absolute risk (\%) & $\begin{array}{c}4.09 \\
(3.91-4.29)\end{array}$ & $\begin{array}{c}2.74 \\
(2.61-2.90)\end{array}$ & $\begin{array}{c}1.89 \\
(1.76-2.02)\end{array}$ & $\begin{array}{c}1.51 \\
(1.41-1.61)\end{array}$ \\
\hline
\end{tabular}

Abbreviations: APOE-HDLC/HDLC ratio, the ratio of apolipoprotein Econtaining HDL cholesterol to total HDL cholesterol; CI, confidence interval; $\mathrm{F}$, female; HR, hazard ratio; $\mathrm{M}$, male.

$\dagger$ All substantial models are additionally adjusted for sex, age (5 year), BMI, smoking status, SBP (10 mmHg), LDLC (30 mg/dl), DM, high TG ( $\geq 150 \mathrm{mg} / \mathrm{dl}$ ), anti-dyslipidemic medication, and log-transformed hs-CRP. 